\title{
S11_Figure: CD177/CD177P1 Sequence Alignment
}

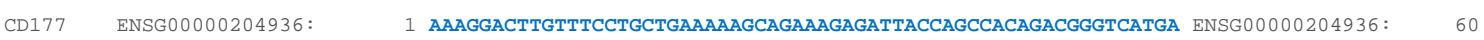

CD177 ENSG00000204936: 61 GCGCGGTATTACTGCTGGCCCTCCTGGGTTCATCCTCCCACTGCCAGGTGAGTGATGAG ENSG00000204936: 120

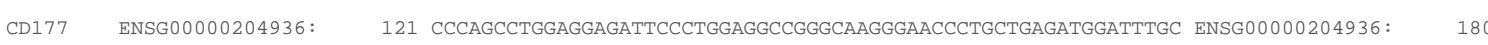

CD177 ENSG00000204936: 181 TCTTGCCACTCCAGGAGTGCAGGCGCTGCTCTGCCAGTTTGGGACAGTTCAGCATGTGTG ENSG00000204936: 240

CD177 ENSG00000204936: 241 GAAGGTGTCCGACCTGCCCCGGCAATGGACCCCTAAGAACACCAGCTGCGACAGCGGCTT ENSG00000204936: 300

CD177 ENSG00000204936: 301 GGGGTGCCAGGACACGTTGATGCTCATTGAGAGCGGTGAGAAGGCCCTGGCGTGCAGAGA ENSG00000204936: 360

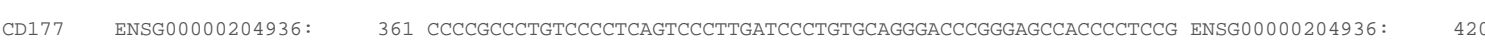

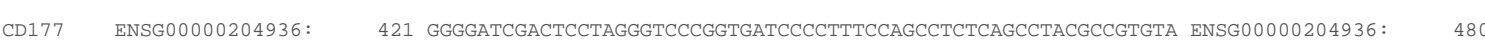

CD177 ENSG00000204936: 481 GCAGCGTCTCCCTCCAGGACCCTGGAGGCCTGACCTCCATCCTCGCTTGCCTCCCTCTTT ENSG00000204936: 540

CD177 ENSG00000204936: 541 CGGTCCAGGACCCCAAGTGAGCCTGGTGCTCTCCAAGGGCTGCACGGAGGCAAGGACCA ENSG00000204936: 600

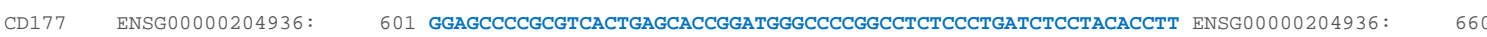

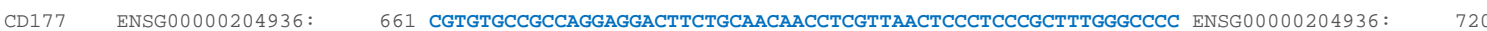

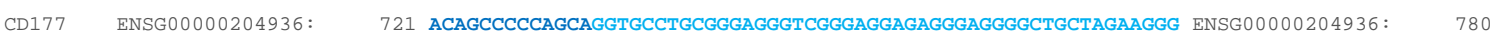

$\begin{array}{llll}\text { CD177 ENSG00000204936: } 781 \text { GATCCGCTGAGCACAGAGGGGCTGTTACGGAG } & \text { ENSG00000204936: }\end{array}$

(Alternative 3' acceptor of exon 3)
CD177 ENSG00000204936: 813 TCCCTCCCACCCTCGCTCGCTATCCCGACCCTCGCTGGCTCCATCCCTCCCCTGACTGCT ENSG000000204936:

CD177 ENSG00000204936: 813 TCCCTCCCACCCTCGCTCGCTATCCCGACCCTCGCTGGCTCCATCCCTCCCCTGACTGCT ENSG00000204936: 872

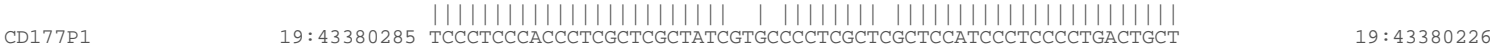

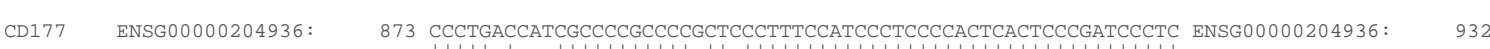

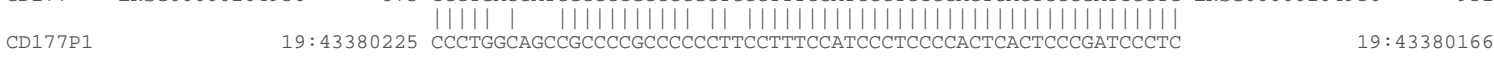

$\begin{array}{llll}\text { CD177 ENSG00000204936: } 933 \text { CCACCGACCT-- } & \text { ENSG00000204936: }\end{array}$

CD177P1 19:43380165 CCACCGACCTCG 19:43380154

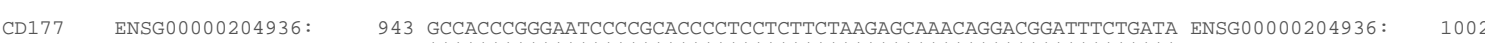
CD177P1 $19: 43380153$ GCCACCCGGGATCCCCGCACCCCCCTCTTCTAAGAGCAAACAGGACGGATTCTGATA
19:43380094

CD177 ENSG00000204936: 1003 TGAAATCACCATTAACTGGGTTATCCTGCATTCTTTTTTTATTCTGTTGCGTTTGGTTCA ENSG00000204936: 1062

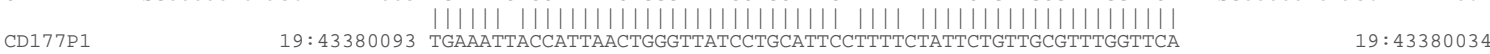

CD177 ENSG00000204936: 1063 AGAAGTTCTCCTGCAGGGTTTTGCTATTTCCTCTTCCTAAGGGAGACTGAACTGTAACT ENSG00000204936: 1122 CD177P1
$19: 43380033$ AGAAGTCTCCTGCAGGGTTTTGCTGTTTCCTCTTCCTAAGGGAGACTGACTGTACT

CD177 ENSG00000204936: 1123 TTCCACTTTCCTTGTCTGGTGTTATAAAGAGTCTAGGGAGATATTTTGCGCTTCTTTTAT ENSG00000204936: 1182 CD177P1
$19: 43379973$ TTCCACTTTCCTTGTCTGGGTTATAAAGAGTCTAGGGAGATATTTGCGCTTCTTTTAT

CD177 ENSG00000204936: 1183 TGGAACAGCTTGTGAACACAGAAGTCTCCTGTTCTTGGAGGTTTAGTAGCGCTTGCAGGT ENSG00000204936: 1242 ||||||||||||||||||||||||||||||||||||||||||||||||| ||||||||| |||||||||| CD177P1 19:43379913 TGGACAGCTTGTGAACACAGAAGTCTCCTGTTCTTGGAGGCTTAGTAGCACTTGCAGGT 19:43379854

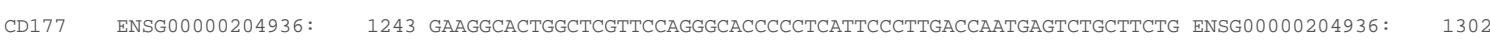
CD177P1 $19: 43379853$ GAAGCACTGGCTCGTTCAGGCACCCCCTCATTCCCTTGACCAATGATCTGCTTCTG
19:43379794

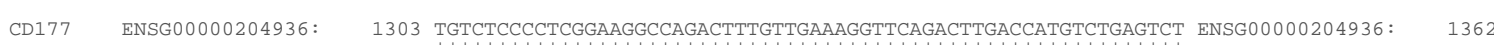

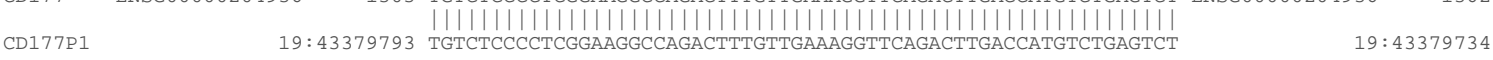
CD177 ENSG00000204936: 1363 ACATTTGGCCTCAGCAGGCCCCTCCTCCACCCTGCCCACCCCCCACCCCCACTTGCCACC ENSG00000204936: 1422 CD177P1 $19: 43379733$ ACATTTGCCTCAGCAGCCCCTCCTCCACCCTGCCCACCCCCACCCCCACTTGCCCC
19:43379674

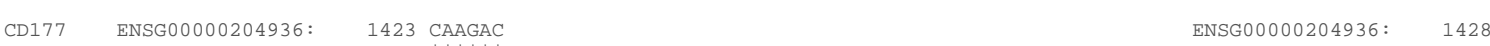

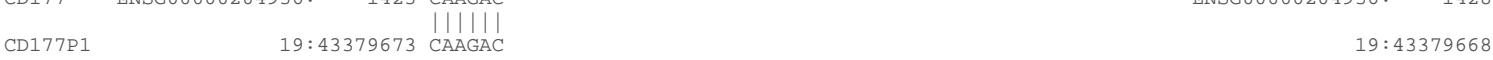
CD177 ENSG00000204936: 1429 CAAGACACCCCCCCTTTTCTTTTCTTTTTTTTT 1461 CD177P1 19:43379667_ III III

CD177 ENSG00000204936: 1462 TTTTTTTTTTCTGAGAAGCGTCTCGCTGTGTCGACCAGGCTGGAGTGCAGTGGCGCAAT ENSG00000204936: 1521 CD177P1 19:43379660 TTTTTTTTTCTGAGAAGCGTCTCGCTGTGTTGACAGGCTAGAGTGCAGTGGGCAAT
19:43379601

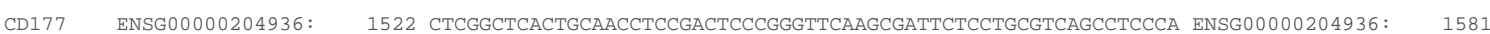

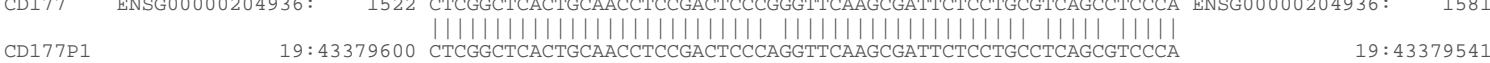
CD177P1 19:43379600 CTCGGCTCACTGCAACCTCCGACTCCCAGGTTCAAGCGATTCTCCTGCCTCAGCGTCCCA 19:43379541

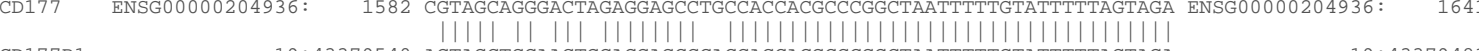
CD177P1 19:43379540 AGTAGCTGGAACTGGAGGAGCCCACCACCACGCCCGGCTAATTTTTGTATTTTAGTAGA 19:43379481

CD177 ENSG00000204936: 1642 GACAGGGTTTCACTATGTTGCCCAGGCTGGTCTTGAACTCCTGACCTCGTGATCCACCTG ENSG00000204936: 1701 CD177P1 $19: 43379480$ GACAGAGTTTCACTATGTTGCCAGGCTGGTCTTGATTCCTGACCTCGTGATCCATCTG
$19: 43379421$ CD177 ENSG00000204936: 1702 CCTCAGCCTCCCAAAGTGTGGGATTACAGGTGTGAGTCACCACACCCAGCCAAGACCAC ENSG00000204936: 1761 CD177P1 190.43379420 CCTCAGCCTCCCAAGTGCTGGGATACAGGTGGAGCCACCACACCCAGCCAAGACCAC
19:43379361 
CD177 ENSG00000204936: 1762 TTTCTGTACCCAGCCCTGGGTGCCTCTACCCAGACCTCCTGTTCATTCTCCCTTCCTCTT ENSG00000204936: 1821 CD177P1 19:43379360 || || || || || || || || || || || || || || || || || || || || || || ||| || || || || ||

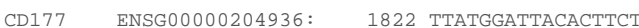

CD177P1 19:43379300 TCATGGGTTACAC -...

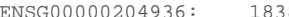

(Alternative $5^{\prime}$ donor of exon 4 )

CD177 ENSGO0000204936: 1839 TTATTTTCCCACATCCAGATCCCATGATGGGAGCTGCAGAAGGCCCCTTTGGGAAGGCTG ENSGO0000204936: 1898 CD177P1 $19: 43379287$ TTATTTTCCCACATCCAGATCCCATGATGGGAGTGCAGAAGGCCCTTTGGAAGCTG
19:43379228

CD177 ENSG00000204936: 1899 AGCAGGTTGACTCTTGAGGCCAGCAAAAGTGGGGTGCAGAGAAGGTATCTGATCAAATCC ENSG00000204936: 1958 1899 AGCAGGTTGACTCTTGAGGCAGCAAAAGTGGGGTGCAGAGAAGGTATCTGATCAAATCC ENSG00000204936: 1958

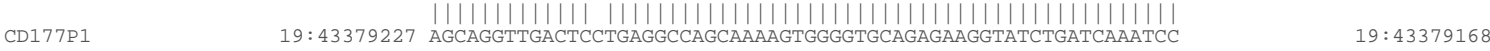
CD177 ENSG00000204936: 1959 AGTGCCCTCTCAGTGCCCCCTCACTCTGTCTGTCACTTTCCTAGACCCAGGATCCTTGAG ENSG00000204936: 2018

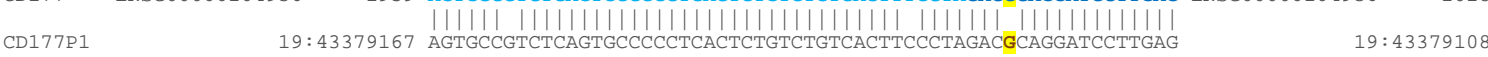
CD177 ENSG00000204936: 2019 GTGCCCAGTCTGCTTGTCTATGGAAGGCTGTCTGGAGGGGACAACAGAAGAGATCTGCCC ENSG00000204936: 2078 CD177P1
19:43379107 GTGCCCAGTCTGCTTGTCTAGGAAGGTGTCTGGAGGGGACACAGAGAGATCTGCCC

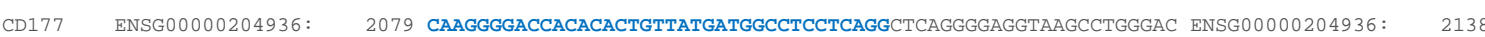
CD177P1 19:43379047 CAAGGGGCCACACACTGTATGATGGCCTCCTCAGGCTCAGGGGGGTAGCCTGGGC
19:43378988 CD177 ENSG00000204936: 2139 ATCGGGGTCCCTGTGGGACTGAACTGGAAGGTCTGGGGACTGAGATCTTAGGCTTTGGG ENSG00000204936: 2198 19:43378987 ATCGGGGCCCTGCGGGACTGAACTGGAAGTCTGGGGACTGAGATCTTAGTCTTGGG CD177 ENSG00000204936: 2199 GAGTGA-

ENSG00000204936: 2204 CD177P1 19:43378927 IIIIII GAGTAG 19:43378921

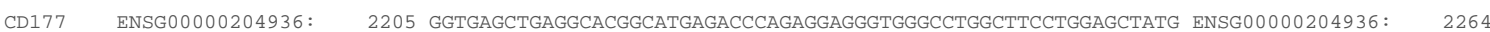
||||||||||||||||||||||||||||||||||||||| ||||||||||||||||||||||||||||||| || CD177P1 19:43378920 GGTGAGCTGAGGCACGGCATGAGACCCAGAGGATGGTGGGCCTGGCTTCCTGGAGCTATG 19:43378861 CD177 ENSG00000204936: 2265 сCTGCCTCTGAGGGTTGGGTGGTCCTGGAGGCAGCATCACTGACTCTCCCTCGCTCCCCC ENSG00000204936: 2324 CD177P1
19:43378860 CCTGCCTCTGAGGTTGGTGGCCTGGAGCAGCATCACTGACTCTCCCTCGCTCCCCC CD177 ENSG00000204936: 2325 TTTCTGCAGGAGGCATCTTCTCCAATCTGAGAGTCCAGGGATGCATGCCCCAGCCAGGTT ENSG00000204936: 2384 $\begin{array}{ll}\text { CD177P1 } 19: 43378800 & \text { TTTCTGCAGGAGCATCTTCTCCAATCTGAGAGTCAGGGATCATGCCCAGCCAGTTT }\end{array}$

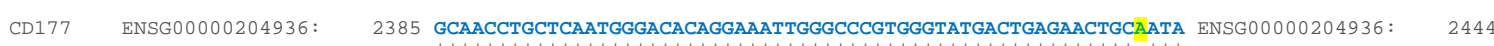
CD177P1
19:43378740 GCAACCTGCTCAATGGACACAGGAATTGGGCCGTGGGTATGACTGAGACTGCGATA CD177 ENSG00000204936: 2445 GGAAAGTGAGTCCTGCCCCTAGGCTGTGCCCTGGACTGCAGCCTCGGGCACGATGACA ENSG00000204936: 2504 CD177P1 19:43378680 TGAAGGTGATCCTGCCCCTAGGCTGTGCCCTGGACTGCAGCCTCGGGCACGATGACA
19:43378621 CD177 ENSG00000204936: 2505 CATGGGGCATCCAGAGCCATCACACCAAAGCCAGCAGGAGGAAGGTCAAGGGTGCAGGGT ENSG00000204936: 2564 CD177P1 $19: 43378620$ CATGGGGATCCAGAGCCATCACACCAAGCCAGCAGGAGAGGTCAGGGTGAGG
19:43378561

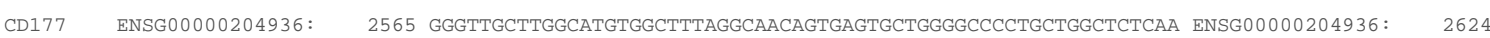

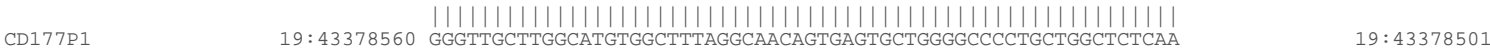

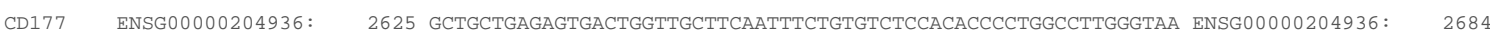

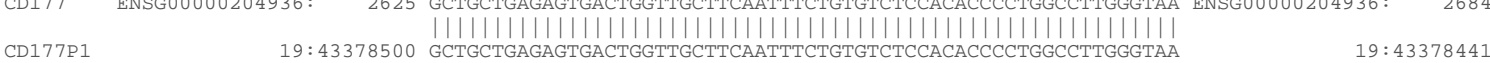
CD177P1 19:43378500 GCTGCTGAGAGTGACTGGTTGCTTCAATTTCTGGTCTCCACACCCCTGGCCTTGGGTAA 19:43378441 CD177 ENSG00000204936: 2685 CCCTAGGTCTCCCACTTCCCTAGGCCACCGTAGCCTCTCTCCCACCCGTATCAGCCCCG ENSG00000204936: 2744 CD177P1 19:43378440 CCCTAGGTCTCCCACTTCCCTAGGCCACCGTAGCCTCTCTCCCACCCGTATCAGCCCCCG 19:43378381

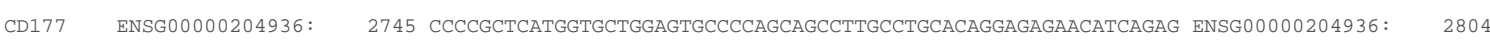
CD177P1 19:43378380 CCCCGCTCATGGTGTGGAGTCCCCAGCAGCCTTGCCTGCACAGGAGAGACATCAGAG CD177 ENSG00000204936: 2805 AGAAAATCAAGGGACTTTGGGTGTGACAGAGAAAGCAAGAGTTAAAGGAGGACACTGAG ENSG00000204936: 2864

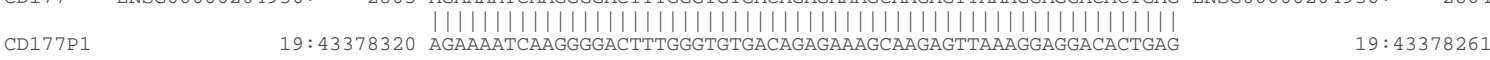

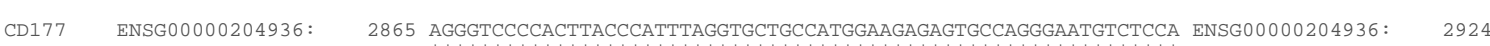
CD177P1
$19: 43378260$ AGGGTCCCCACTTACCCATTTAGGTGCTGCCATGGAAGAGAGTGCAGGGATGTCTCCA CD177 ENSG00000204936: 2925 TAAGAAGGGCTCCAAAGCAACAGGAAGCCATGGTGGGGAGGTGGGGGTCTTATAGGAATC ENSG00000204936: 2984 CD177P1 $19: 43378200$ TAAGAAGGCTCCAAAGAACAGGAAGCCATGGTGGGAGGTGGGGTCTATAGGATC
19:43378141 CD177 ENSG00000204936: 2985 CCAGCTTCCCTCCTAGAAGCAAAGTCTTCCCTGCCCCTGCCTCAGTTTCCTTGTCTGCCC ENSG00000204936: 3044 CD177P1 1 | 1|||||||||||||||||||||||||||||||||||||||||||||||||||||| $\mid$
$19: 43378140$ CCAGCTTCCCTCCTAGAGCAAGTCTTCCTGCCCTGCCTCAGTTCCTTGCTGCCC

CD177 ENSG00000204936: 3045 ACATCACGCTAACCCTTAGAGCTTAGAAGAGAGAGTTGTGTGAGCTCTGCACACATTCCT ENSG00000204936: 3104 CD177P1
19:43378080 ACATCACGCTAACCCTTAGAGCTTAGAGAGAGAGTTGGTGAGCTCTGCACACATTCCT CD177 ENSG00000204936: 3105 CTGTGTATTTGGCTGTTTCTCCCTAGCTAGGCTGGGAGCCTGAGGCTAGGGGTTGGTAC ENSG00000204936: 3164

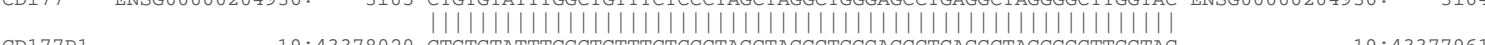


CD177 ENSG00000204936: 3165 CACTGGCACCAGGTTTCCAGCCTTGGGTAGCTCAGGAGACGGCTCAGGAAGGGTACCAG ENSG00000204936: 3224 CD177P1 $19: 43377960$ CACTGGCACCAGGTTCCAGCCTTGGTAGCTCAGGAGACGCTCAGGAGGGACCAG
19:43377901

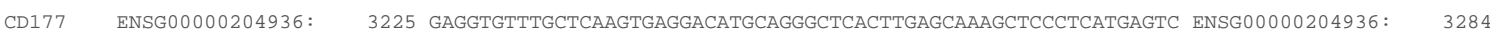
||||||||||||||||||||||||||||||||||||||||||||||||||||||||||||||||| CD177P1 19:43377900 GAGGTGTTTGCTCAAGTGAGGACATGCAGGGCTCACTTGAGCAAAGCTCCCTCATGAGTC 19:43377841 CD177 ENSG00000204936: 3285 AGACCCTGTTTCTACCTGAAGGGCCTCCAGGACTAGGAGGGGAGGTGCTACAGACACAG ENSG00000204936: 3344 CD177P1
19:43377840 AGACCCTGTTCTACCTGAGGGCTCCAGGACTAGGAGGGGAGGTCTACAGACACAG

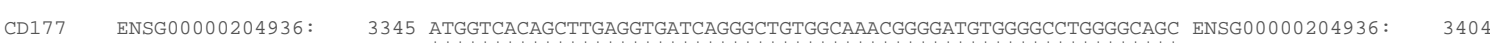
CD177P1 19:43377780 ATGGTCACAGCTTGAGTGATCAGGGCTGTGGCAACGGGGTGTGGGCCTGGGCAGC CD177 ENSG00000204936: 3405 TCAGAGGAGGAAATACTGATGTTGTTGGGGTAGAGAGTCAAGGAGGGCTTTACAGAGGA ENSG00000204936: 3464 CD177P1
$19: 43377720$ TCAGAGGAGGAAATACTGATTTGTGGGGTAGAGAGTAAGGAGGCTTACAGAGGA CD177 ENSG00000204936: 3465 GGTGGCCTGTGAACCGTGTGTTAAAGATGACCTACAGCTTATTGGTAGAAGAGGAAGAGG ENSG00000204936: 3524 CD177P1 $19: 43377660$ GGTGGCCTGTGACCGTGTTTAAAGATGACCTACAGCTTATTGGTAGAGAGGAGAGG
19:43377601 CD177 ENSG00000204936: 3525 ACATTGGTGGCAGAGGGACAGTGTGTAGCAGCTGCATAGGCCTGGAGGTGGAGTGAGC ENSG00000204936: 3584

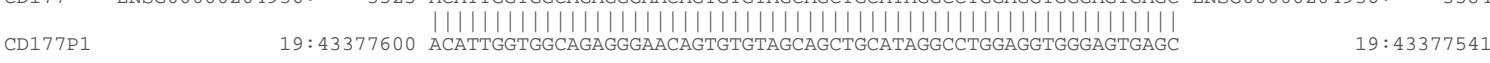
CD177 ENSG00000204936: 3585 CTGCTGTTTTCAAGAAGAGTCCCTCACTCCACCTGAGAAGGACTCATCAAGATGAGGGGG ENSG00000204936: 3644

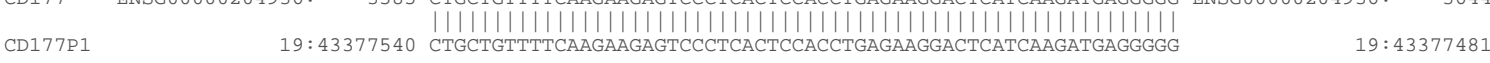

CD177 ENSG00000204936: 3645 TACAGCTGGGATTGGGGTGATTCGAAGTTTTCAGGTGGACAAGGTGGTGTGGGGTCG ENSG00000204936: 3704

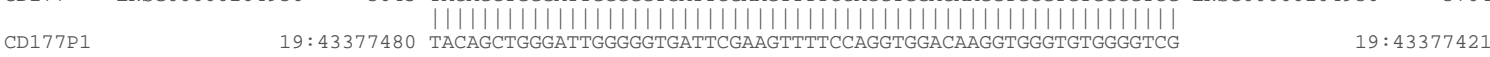

CD177 ENSG00000204936: 3705 AAGAGGGAGCTGCTCCAAGCCAAAGGCACAGCAGGTGTGAGGCTGAGGAGGAATGAACT ENSG00000204936: 3764 CD177P1
$19: 43377420$ AAGAGGGAGCTGCTCCAACCAAAGGCACAGCAGGTGGAGGCTGAGGAGAAATAACT CD177P1 19:43377420 AAGAGGGAGCTGCTCCAAGCCAAAGGCACAGCAGGTGTGAGGCTGAGGAGGAAATGAACT 19:43377361 CD177 ENSG00000204936: 3765 GGCATGCCTTCAGAATGGATACTGGTGGGGGAGGAGAGTTAAGTGGGAACCTGGTGGT ENSG00000204936: 3824 CD177P1 19:43377360 GGCATGCCTTCAGAATGGATACTGGTGGGGGGGAGAGTTAAGTGGGAACCTGGTGGT 19:43377301 CD177 ENSG00000204936: 3825 CGCTCAAGTACCTGAAACCCAGGCTGAGGGGTGACCCTCTCCTGAAGGCAGGGGAGCC ENSG00000204936: 3884

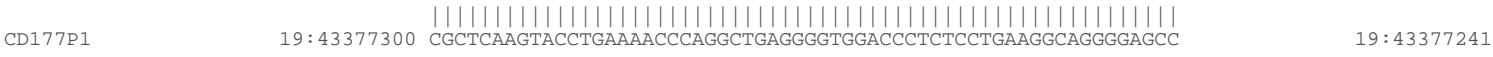
CD177 ENSG00000204936: 3885 ATGGAAGATTGTGGAGCAGGGAGGGCAGGGTCTGAGCCATTTATCTTTCCTTTTGACTGA ENSG00000204936: 3944 CD177P1 $19: 43377240$ ATGGAAGATTGTGAGCAGGAGGGCAGGTCTGAGCCATTTATCTTTCCTTTTGACTGA
19:43377181 CD177 ENSG00000204936: 3945 TGCTGCTGTTAAGGATCTGGCCCTGGTCTCTGGGACCAGATGACACTGATAACCCAGAAG ENSG00000204936: 4004 CD177P1 $19: 43377180$ TGCTGCTGTTAGGATCTGGCCTGGTCTCTGGGACCAGATGACACTGATAACCCAGAAG
19:43377121 CD177 ENSG00000204936: 4005 GTATTTCAGAAACAAATCAGACGCAACACATGCCACACACACAGCCCACAATAAGCACAG ENSG00000204936: 4064

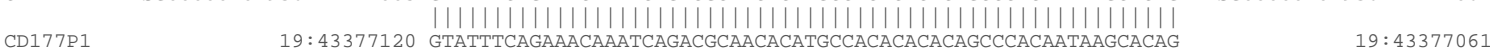
CD177 ENSG00000204936: 4065 CAGACACGAATGCGTCACACCATGACACATACACACCACACATCACATACCACACACACA ENSG00000204936: 4124 CD177P1 19:43377060 CAGACACGATGCGTCACACCATGACACATACACACCACACATCACATACCACACACACA

CD177 ENSG00000204936: 4125 CTCCCCCATACCCCTGACATACCACCTATGGACACAAATCACACACAGAGCACACAATA ENSG00000204936: 4184

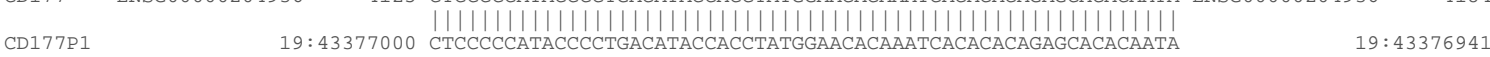

CD177 ENSG00000204936: 4185 TGCACAACACACACAAATGCATCACACGAGTGCACAATTACTACGTGTACATGTAGTGTG ENSG00000204936: 4244

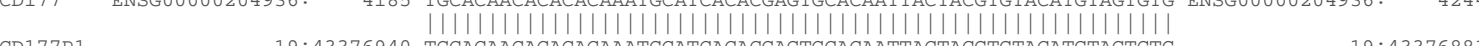
CD177P1 19:43376881

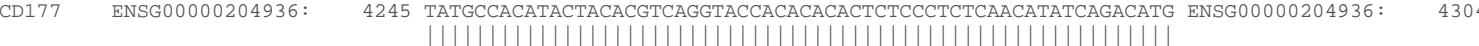
CD177P1 $19: 43376880$ TATGCCACATACTACACGTCAGGTACCACACACACTCTCCCTCTCAACATATCAGACATG
$19: 43376821$ CD177 ENSG00000204936: 4305 CAATACATACCACACACAACACACAATACACACAACACATCTGGCATGCCACACACACCA ENSG00000204936: 4364 CD177P1 $19: 43376820$ CAATACATACCACACACAACACACAATACACACACACATCTGGCATGCCACACACACCA
19:43376761 CD177 ENSG00000204936: 4365 CATGCAAGCAAAACACAGATACACAGAACACACACCACACCAAACACACAAAACAAACAC ENSG00000204936: 4424 CD177P1 $19: 43376760$ CATGCAAGCAAAACACAGATACACAGACACACACCACACCAAACACACAAACAAACAC
19:43376701 CD177 ENSG00000204936: 4425 ATACCACATATATCACACACAGTATGTCTACACATGCACGCACCACACAAACATACCATG ENSG00000204936: 4484 CD177P1 190.43376700 ATACCACATATATCACACACAGTATGTCTACACATGCACGCACCACACAACATACCATG
19:4 CD177 ENSG00000204936: 4485 AACACACCACACACCCCATACAACCACACCCCCCACATGTATACTACGCACTCACACACA ENSG00000204936: 4544 CD177P1 19:43376640 AACACACCACACACCCCATACAACCACACCCCCCACATGTATACTACGCACTCACACACA
19:43376581 CD177 ENSG00000204936: 4545 GCACACATACCTGCACACATCACGCATGTGCACACACTACACAAACGCACCACATAAAAC ENSG00000204936: 4604

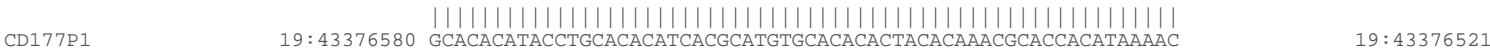
$\begin{array}{lllll}\text { CD177 ENSG00000204936: } 4605 \text { ACAGCGACTATGCACCAATCAGTGCTGTTCATAGAGATTCA- - - } & \text { ENSG00000204936: }\end{array}$ CD177P1
19:43376520 ACAGCGACTATGCACCAATCAGTGCTGTTCATAGAGATTCATCT

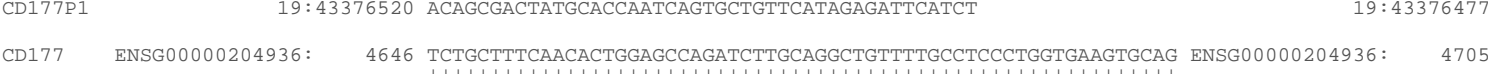

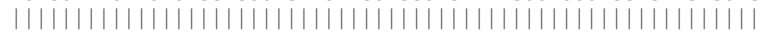


CD177 ENSG00000204936: 4706 TTCAGCAGTTTAGCAGACAGTGAGCATCACAAGACCAGGCATCAGAAACTGGGGGACAA ENSG00000204936: 4765 CD177P1 19:43376416 ITCAGCAGTTAGCAGACAGTGAGCATCACAAGACCAGGCATCAGAACTGGGGACAA

CD177 ENSG00000204936: 4766 CAAGACGTTGGGACCAAGTGCAGGAGAGAAGAGGCTTCAGGGTGATGGAACCGCTTATA ENSG00000204936: 4825 ||||||||||||||||||||||||||||||||||||||||||||||||||||||||||||||||||||||||||| 19:43376297 CD177 ENSG00000204936: 4826 AGAAGCCGCAGCAGTGGGAGGCTGGGTGCAAGGATGCAGGTTACTAAAGGGGCCATGG ENSG00000204936: 4885 CD177P1
19:43376296 AGAGCCGCAGCAGTGGAGGCTGGGTGAAGGATGAGGTACTAAAGGGCCATGG CD177 ENSG00000204936: 4886 CAGCAGCCCCTTGTGAAGAACCAAGGGGCCCGGGCAGATAAGGCCCTGGCTGCCCCTAGA ENSG00000204936: 4945 CD177P1 $19: 43376236$ CAGCAGCCCCTTGTGAGACCAAGGGCCCGGGCAGATAAGGCCTGGCTGCCCCTAGA
19:43376177 CD177 ENSG00000204936: 4946 GGAGCTGGGCAGCGGTCCCAGTGAGAGAGCAGGGAAGAGCCCACGTCTGAATCAGGGACA ENSG00000204936: 5005

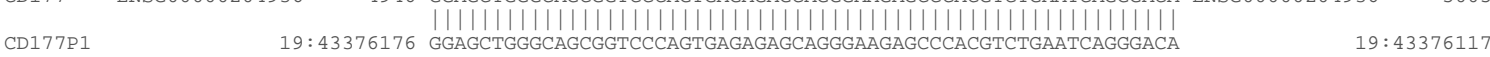
CD177 ENSG00000204936: 5006 CGTGGGTCCCAGGCCAGCTCTGCCGCCGAAACAGCTTTCCAGCCTCAGCTTCACACGAG ENSG00000204936: 5065 CD177P1 19:43376116 CGTGGGTCCAGGCCAGCTCTGCCGCCGAACAGCTTTCCAGCCTCAGCTTCACACGA
19:43376057 CD177 ENSG00000204936: 5066 GATCAAAAGTCTCCATATCTCATGGAGCCTCTTTCCCTGGATTTGGGAAGAGGAGGGGA ENSG00000204936: 5125

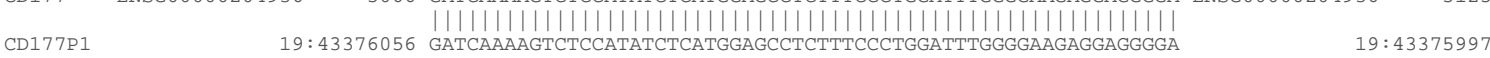
CD177 ENSG00000204936: 5126 TGGTTGCAGACATGGGACGGAAGCAGATGCAAAGACCATGATGTGGGTGAGTGCCTGCCA ENSG00000204936: 5185 CD177P1 19:43375996 TGGTTGCAGACATGGGACGAAGCAGATGCAAGACCATGATGTGGTGAGTCCTGCCA
19:43375937

CD177 ENSG00000204936: 5186 GAAACACCTGTGCGGAGCAGAGTGATGGAGGGAGCAGGGAGGCGGCCGCGTCTTATCAGG ENSG00000204936: 5245 |||||||||||||||||||||||||||||||||||||||||||||||||||||||||||||||||||||| CD177P1 19:43375877

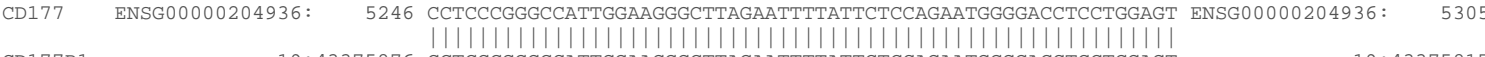
CD177P1 19:43375876 CCTCCCGGGCATTGGAAGGCTTAGAATTTTATTCTCCAGAATGGGACCTCCTGGAGT 19:43375817

CD177 ENSG00000204936: 5306 CTTCGGAGCAGAGGAGTGACATGAACTGACTTAGGTTTACCTAGCCTCCCTCTGCCTGCT ENSG00000204936: 5365 CD177P1
19:43375816 CTTCGGAGCAGAGGAGTACATGAACTGACTTAGGTTACCTAGCCTCCCTCTGCCTGCT CD177 ENSG00000204936: 5366 GGGTGGAGAATGGAGCGAGAGGAACAGTGACAGGCGGGTGCAGGGATTGCCCCAGGAGTC ENSG00000204936: 5425

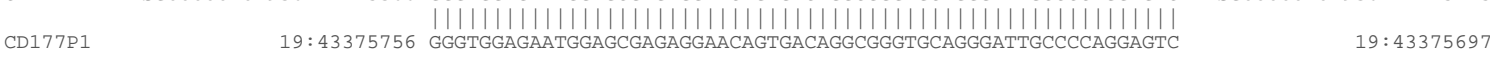

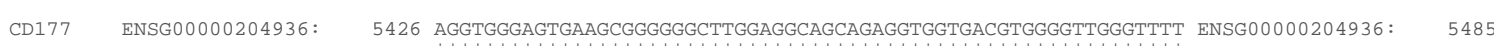
CD177P1
19:43375696 AGGTGGGAGTGAGCGGGGGCTTGGAGGAGCAGAGGTGGTGAGTGGGTTGGGTTT CD177 ENSG00000204936: 5486 GAAGATTTTCTGCAGCTCAAGCCAGTAGGATTACTTGAGGAACACAATGTGGGAGAGAAA ENSG00000204936: 5545 CD177P1 19:43375636 GAAGATTTTCTGCAGCTCAACCAGTAGGATTACTTGAGGACACAATGTGGAGAGAAA
19:43375577 CD177 ENSG00000204936: 5546 GAGCGTGGTCAAGGACAACACCAATGTGTTCAGCTAGACTGGCAGAAACGAGCCGCCACG ENSG00000204936: 5605 CD177P1 $19: 43375576$ GAGCGTGGTCAAGACACACCAATGTGTCAGCTAGACTGGCAGAACGAGCCGCCACG
19:43375517

CD177 ENSG00000204936: 5606 GGTGTAAGTAGGGGAGATGGGAGTGGGAGAGAAGGTGGGAGAAGGAGCAGGAGCCAT ENSG00000204936: 5665 CD177P1 19:43375516 GGTGTAAGTAGGGAGATGGAGTGGGAGAGAGGGTGGAGAAGGAGCAGGAGCAT

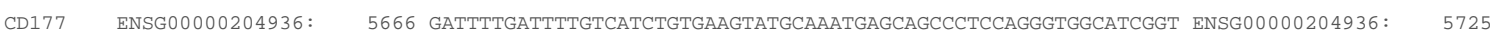
CD177P1
$19: 43375456$ GATTTTGATTTGTCATCTGTGAAGTATGCAAAGAGCAGCCCTCCAGGGGGCATCGGT CD177P1 19:43375456 GATTTGATTH:43375397

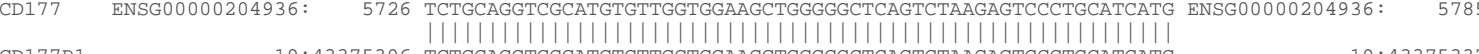
CD177P1 19:43375396 TCTGCAGGTCGCATGTGTTGGTGGAGCTGGGGGCTCAGTCTAAGAGTCCCTGCATCATG_ 19:43375337

CD177 ENSG00000204936: 5786 GGACCGATCCCCCCTGCTCTGTGATGGCCGTGAGGGGAGGCCAGGGGCTGTGGTTTCACA ENSG00000204936: 5845 CD177P1 $19: 43375336$ GGACCGACCCCCCTGCTCTGTGATGCCGTGAGGGAGGCAGGGGTGTGGTTCACA
19:43375277 CD177 ENSG00000204936: 5846 CTAGAGCCTTGAGGGCTCGGGGCCAAGGTAGCCGAGGATGGTCTTCAGTACCTGTCTCC ENSG00000204936: 5905 CD177P1
19:43375276 CTAGAGCCTTGAGGCCTCGGGCCAGGTAGCCGAGATGGTCTTCAGTACCTGTCTCC CD177 ENSG00000204936: 5906 CTCCTGCTCCCAACCCCGACCCTGAGGGTCCAGAGTCCCTGGGTTCCCAGCCTACCTCTG ENSG00000204936: 5965 CD177P1 190.43375216 CTCCTGCTCCCAACCCGACCCTGAGGTCCAGAGTCCCTGGGTCCCAGCCTACCTCTG
19:43375157 CD177 ENSG00000204936: 5966 CCACTCACAAGCTGTGTGGCATGGGCAGCTACTGAGCAATCCTGCCTCTGTTTCCCTTGT ENSG00000204936: 6025 CD177P1 $19: 43375156$ CCACTCACAAGCTGTGTGCATGGGCAGCTACTGAGCAATCCTGCCTCTGTTCCCTTGT
19: $19: 43375097$ CD177 ENSG00000204936: 6026 TGCAAATGAGGGTGTTGATTGTGATCACACCACCTACTGCTGGGGTGCTGTGGGGAATG ENSG00000204936: 6085 CD177P1
19:43375096 IGCAAATGGGGTTGATTGTGATCACACCACCTACTGCTGGGGGCTGTGGGAATG

CD177 ENSG00000204936: 6086 AGGCATCCCAGATAAGCTGTGTTCCCTGGAACCTTAGATGGGTGGATCTGTGGGAAGCAA ENSG00000204936: 6145 CD177P1
19:43375036 AGGCATCCCAGATAAGCTGTGTTCCCTGGACCTTAGATGGGTGGTCTGTGGGAGCAA

CD177 ENSG00000204936: 6146 TGAGGTCAGCCAGGTGCAGAGGCAGTGCTCAGCGGCAGTGCTCAGCCCACCCTGGCCCTT ENSG00000204936: 6205 | 


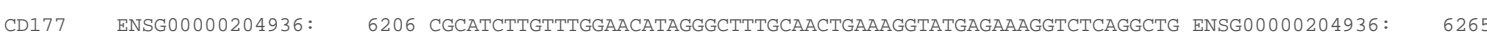
CD177P1 19:43374916 CGCATCTTGTTGGACATAGGGCTTGCACTGAAGGTATGAGAAGGCTCAGGCTG

CD177 ENSG00000204936: 6266 CCTTCCTTATTATTGAAATGAGGACCTTAAAGCTCAGATGGGGAGCAGAGATGGGATGA ENSG00000204936: 6325 | |||||||||||||||||||||||||||||||||||||||||||||||||||||||||||||| || || 19:43374797 CD177 ENSG00000204936: 6326 GGATAAGAAGAGCTTCCATTGCTCTCACAGCTAAAGCAAGGAAGGCCGTTCCCCCAGG ENSG00000204936:

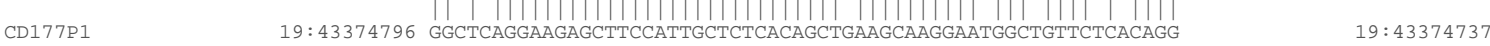
CD177 ENSG00000204936: 6386 GCTGTGAGAGGACGTCCCTGCGGGTGTTCAAGCAGAAACAGGGAACAATGGGGGTGGGAT ENSG00000204936: 6445 CD177P1
19:43374736 GCTGTGAGAGGACGTCCTGCGGTGTTCAAGCAGAACAGGGACAATGGGGTGGG CD177 ENSG00000204936: 6446 TTCGACTCCCAAGTCCCTTCTGAATCCTTGGTTAAGGGAATCTGTGGCCAGGTCACCTGG ENSG00000204936: 6505

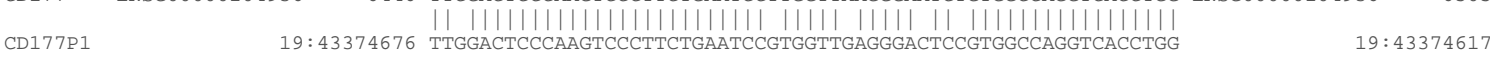
CD177 ENSG00000204936: 6506 AGTGTGACTCAAGAGTGTGATCACCTTCCCTAGCCCAGGACGTGGAGGGACAGACATGGT ENSG00000204936: 6565

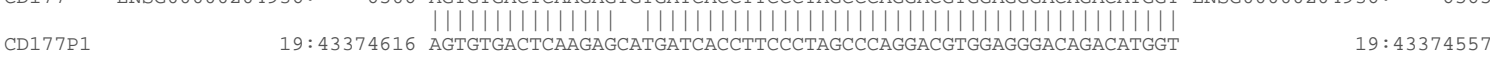
CD177 ENSG00000204936: 6566 GGGTTCCTGGCTTACACACACCCTGGGATTCCTCTCCCCAGATTTTCTGACCTGTCATCG ENSG00000204936: 6625

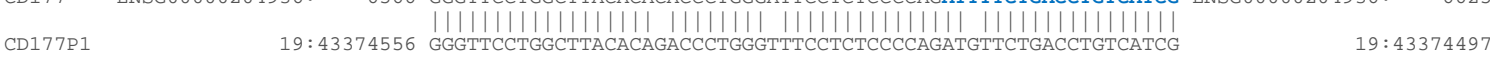
CD177 ENSG00000204936: 6626 GGGGACCACCATTATGACACACGGAAACTTGGCTCAAGAACCCACTGATTGGACCACATC ENSG00000204936: 6685

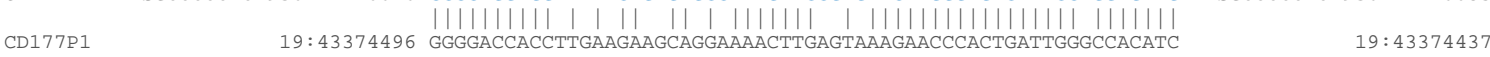
CD177 ENSG00000204936: 6686 GAATACCGAGATGTGCGAGTGGGGCAGGTGTGTCAGGAGACGCTGCTGCTCCTAGATGT ENSG00000204936: 6745

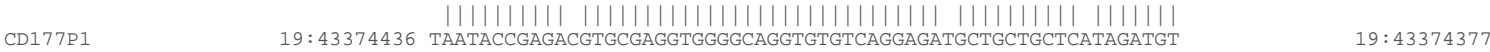

CD177 ENSG00000204936: 6746 AGGTACGTGGACTGAGGTAGAAGACGAACACCTGTCCCAAGTCCCTGGCAGCTCCCTCCA ENSG00000204936: 6805

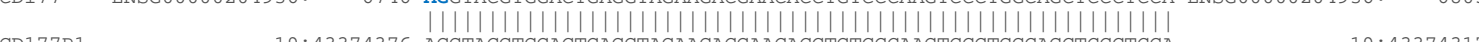
CD177P1 19:43374317

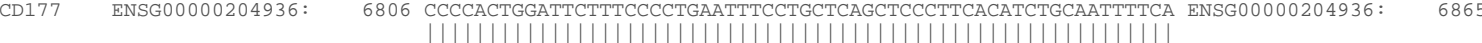

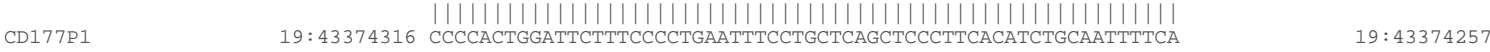
CD177 ENSG00000204936: 6866 ATCCGACTCTTCTTCTCTCTGCATCTTGGGTTCCCCTTATGAAAACTGGGGTGAAGCAG ENSG00000204936: 6925 CD177P1 $19: 43374256$ ATCCGACTCTTCTTCTCTCTGCATCTTGGTTCCCCTATGAAACTGGGGTGAGCAG
19:43374197 CD177 ENSG00000204936: 6926 TGGGAATGACAGTGTCTGCCTTTCTGGGGAGGGGTGACCACGTATGCGGTAATCCCTGCC ENSG00000204936: 6985 CD177P1 $19: 43374196$ TGGGATGACAGTGCTGCCTTTCTGGGAGGGTGACCACGTATGCGGTATCCCTGCC
19:43374137 CD177 ENSG00000204936: 6986 CAGGGTGGAGAACCGTGCCAACACCGAGGACTCTCAGTGGCTTCATACATTCTGGAAATG ENSG00000204936: 7045 CD177P1 $19: 43374136$ CAGGGTGAGACCGTGCAACACCGAGGACTCTCAGTGGCTTCATACATTCTGGAATG
19:43374077 CD177 ENSG00000204936: 7046 TTTATCCAGCATTTGTTTAGGTGCCGCAGATCCAATCCCATCTGTATTCCTGCAGCTTT ENSG00000204936: 7105

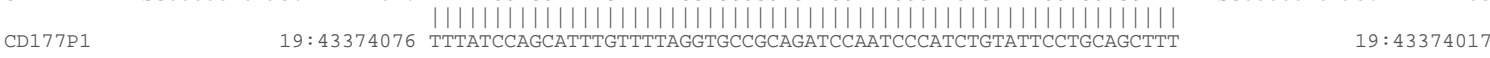
CD177 ENSG00000204936: 7106 ATATGCCTTGAGAAACAGGAAACAAACAAACAAAAGCAGCAAAGTATGGGGAATGGAGA ENSG00000204936: 7165 CD177P1 19:43374016 ATATGCCTTGAGAACAGGAACAAACAACAAAAGCAGCAAGTATGGGATGGAGA

CD177 ENSG00000204936: 7166 TCCCAGCTCTGCAGAGAATCTGGCAAGGAAAAGGAGATAGGAAAAGTGAGGGGAAGGCT ENSG00000204936: 7225 CD177P1
19:43373956 TCCCAGCTCTGGAGAGAATCTGGCAAGGAAAGGAGATAGGAAAGTGAGGGAGGCT CD177 ENSG00000204936: 7226 GCAATTTTAAGCTCAGTGCTCAGGGGAGACTCATGGAGAAGGTGACCTTTGAGCGAGAAC ENSG00000204936: 7285 ||||||||||||||||||||||||||||||||||||||||||||||||||||| ||||||||||||||| CD177P1 19:43373896 GCAATTTAAGCTCAGTGCTCAGGGGAGACTCATGGAGAAGGTGACGTTTGAGCGAGAAC 19:43373837 CD177 ENSG00000204936: 7286 TGGGAGGAGGCAGAGCTTCGGGCAGGACCCAGGCCCCAGCCTCCGTCTTTGCCCCACACT ENSG00000204936: 7345

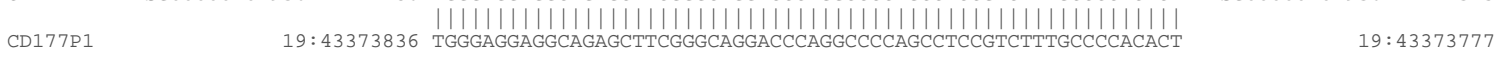
CD177 ENSG00000204936: 7346 AAACATGACCCAGCAGTTGTGATCAGGGCATTCACCCTCTGCCTGGGGGTATTGTGAAG ENSG00000204936: 7405 CD177P1
19:43373776 AAACATGACCCAGCAGTGTGATCAGGGATTCACCCTCTGCCTGGGGGTATGTGAG CD177 ENSG00000204936: 7406 GGCAGGGAGTCCAGCTCTGGAGCCCCTGCTGCCCTGGGAGCTGCCCAGTCCCCAGCCCAG ENSG00000204936: 7465

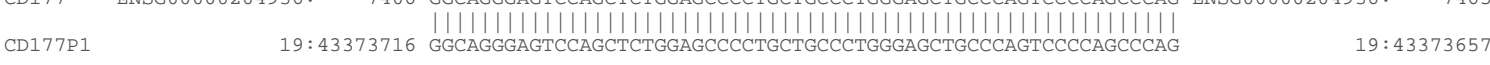
CD177 ENSG00000204936: 7466 CTTTCCCTCTCACCCTCAGGACTCACATCAACCCTGGTGGGGACAAAAGGCTGCAGCACT ENSG00000204936: 7525 $\begin{array}{lll}\text { CD177P1 } & \text { 19:43373656 CTTTCCCTCTCACCCTCAGGACTCACATCACCCTGGTGGGACCTAAGCTGCAGCGCT }\end{array}$ CD177 ENSG00000204936: 7526 GTTGGGGCTCAAAATTCCCAGAAGACCACCATCCACTCAGCCCCTCCTGGGTGCTTGTG ENSG00000204936: 7585

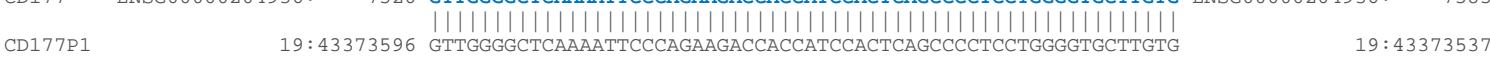
CD177 ENSG00000204936: 7586 GCCTCCTATACCCACTTCTGCTCCTCGGACCTGTGCAATAGTGCCAGCAGCAGCAGCGTT ENSG00000204936: 7645

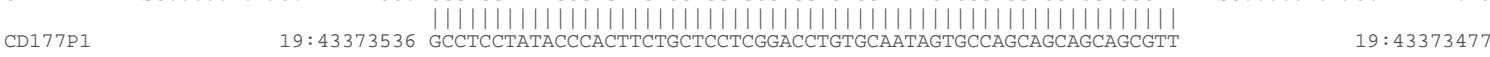

CD177 ENSG00000204936: 7646 стGCTGAACTCCCTCCTCCTCAAGGTATGGGATCCAGGGCCGTGGAGAATGAGGCCCA ENSG00000204936: 7705 CD177P1
$19: 43373476$ CTGCTGAACTCCCTCCCTCCTCAAGGTATGGGATCCAGGGCCGTGGAGAATGAGGCCA $\begin{array}{lll}\text { CD177P1 19:43373476 CTGCTGAACTCCCTCCCTCCTCAAGGTATGGGATCCAGGGCCGTGGAGAAATGAGGCCCA } & 19: 43373417 \\ \text { CD177 ENSG00000204936: } 7706 \text { GACACACAGAGACTCTGGCCCAAGGTGGCCAGCTGCTCTGAGCACAAAGTCATGTACCCC ENSG00000204936: } & 7765\end{array}$ |||||||||||||||||||||||||||||||||||||||||||||||||||||||||||| 


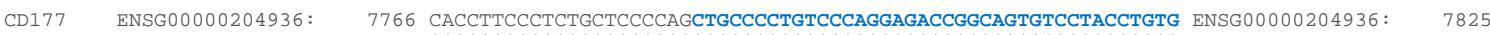

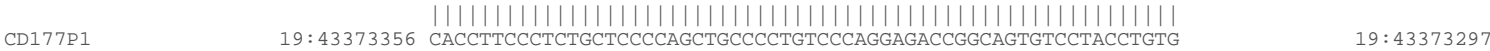

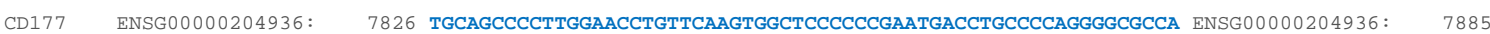

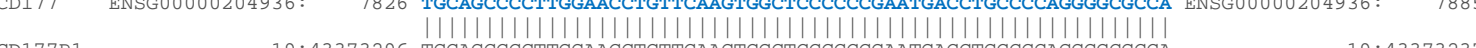
CD177 ENSG00000204936: 7886 CTCATTGTTATGATGGGTACATTCATCTCTCAGGAGGTGAGTGCTGCAAGCAGGGCCCCA ENSG00000204936: 7945 CD177P1 $19: 43373236$ CTCATTGTTATGATGGTACATTCATCTCTCAGGAGTGAGTGCTGCAAGCAGGCCCCA
19:43373177 CD177 ENSG00000204936: 7946 AGGATGAAGGCACTGGTGGCCTGGACTCCTGGGTCTGAGGGAGGAGGGGCTGGGGGCCTG ENSG00000204936: 8005 CD177P1
19:43373176 AGGATGAAGGCGTGGTTCCTGGGCTCCTGGGACTGAGGGAGAAGGGCTGGGGCCTG $\begin{array}{llll}\text { CD177 ENSG00000204936: } 8006 \text { GACTCCTGGTCCGAGGAGGAGGCGCTGGGGGCCT } & 8040\end{array}$ CD177P1 19:43373116 IIIIIIIII

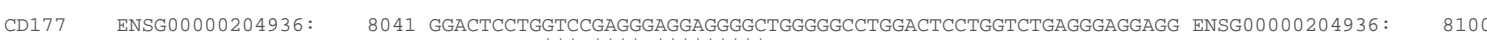
CD177P1 19:43373118 $\quad$ || 1|||||||||||| $\mid$ |

CD177 ENSG00000204936: 8101 CGCTGGGGCCTGGGCTCCTGGTCCCAGGGAGGAGGGCTGGGTGCCTGGACTCCTGGTC ENSG000000204936: 8160 CD177 ENSG00000204936: 8161 TGAGGGAGGAGGGCTGGGGGCCGGGGCTCCTGGGTCTGAGGAGCTGAGGCTCTGGACTC ENSG00000204936: 8220 CD177 ENSG00000204936: 8221 CTGGGTCTGAGGTAGGAGGGACTGGGGGCTGGACTCCTGGGTCTGAGGGAGGAGGGGCT ENSG00000204936: 8280 $\begin{array}{llll}\text { CD177 ENSG00000204936: } 8281 \text { GGGGGCCTGGACCCCTGGGTCTGAGGAGCTGGA } & \text { ENSG00000204936: } 8313\end{array}$

CD177 ENSG00000204936: 8314 GCTGGGGGTCTGGGCTCCTCGGTCTGAGGGAGGAGGGTCTGGGGCCTGGACTCCTGGGTT ENSG00000204936: 8373 ||||||||||||| |||||| || ||||||||||||||||||||||||||||||||||||||||| 3091 GCTGGGGGTCTGAGCTCCTGCGTTTGAGGGAGGAGGGTCTGGGGC

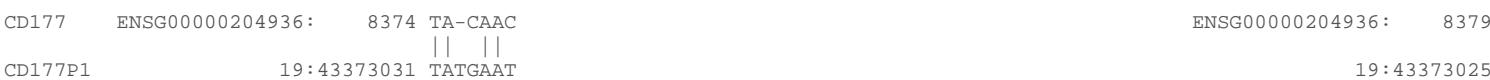

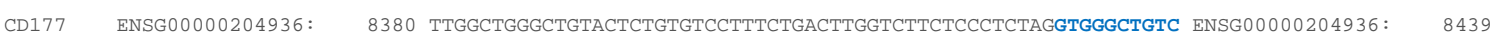

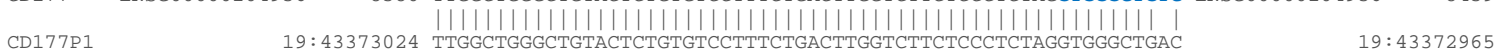

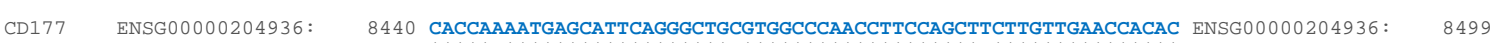
CD177P1 $19: 43372964$ CACCAGAATGAGATTCAGGCTGTGTGGCCACCTTCCAGCTCCTTGTTGACCACAC
19:43372905

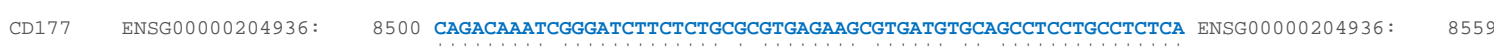
CD177P1
19:43372904 CAGACAAATGGGATCTTCTCTGTGTGTGAGAGGGTGATAGCCGCCTCCTGCCTCTCA

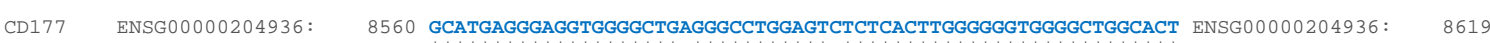
CD177P1 $19: 43372844$ GCATGAGGGGGTGGGCTGGGGGCTGGAGTGTCTCACTTGGGGGTGGGGCTGGACT
19:43372785 CD177 ENSG00000204936: 8620 GGCCCCAGCGCTGTGGTGGGGAGTGGTTTGCCCTTCCTGCTAACTCTATTACCCCCACGA ENSG00000204936: 8679

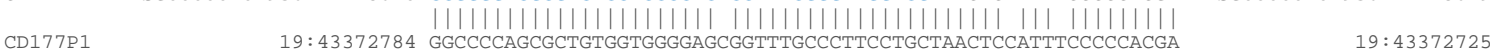

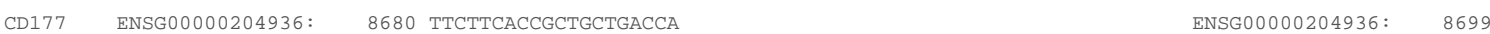
CD177P1 19:43372724 I|| IIIII||||||||| || || $19: 43372705$

CD177 ENSG00000204936: 8700 CCCACACTCAACC-TCCCTCTGACCTCATAACCTAATGGCCTTGGACACCAGATTCTTT- ENSG00000204936: 8757 19:43372704 CC-ACACTCAACCGTCCCTGTGCTTG-TAACCTAATGCCTTGGACACCAGATATTC
CD177P1 CD177P1 19:43372704 CC-ACACTCAACCGTCCTTGTGCTT-TAACCTAATGGCCTTGGACACCAGATTATTTC 19:43372647

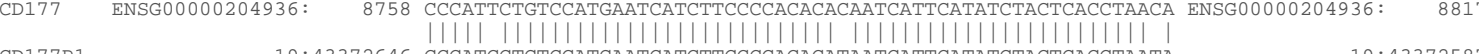
CD177P1 19:43372646 CCCATCCTGTCCATGAATCATCTTCCCCACACATAATCATTCATATCTACTCACCTAATA 19:43372587 CD177 ENSG00000204936: 8818 GCAACACTGGGGAGAGCCTGGAGCATCCGGACTTGCCCTATGGGAGAGGGACGCTGGAG ENSG00000204936: 8877 CD177P1 19:43372586 GCACACTGGGGAGCCTGGAGCAGCCGGACTTGCCCTGTGGGAGAGGGACACTGGA

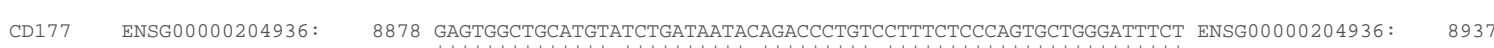
CD177P1 $19: 43372526$ GAGTGGCTGCATGTGCTGATAATAAAGACCCTGTGCTTTCTCCCAGTGCTGGATTCT
19:43372467

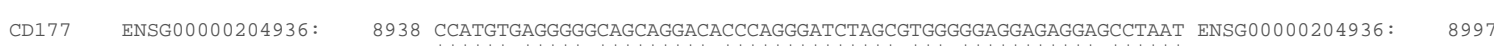
CD177P1
$19: 43372466$ CCATGTAAGGGACAGCAGGACGCCAGGGATCTAGCATGGTGGAGAGAGGACCTAAT CD177 ENSG00000204936: 8998 GAGAAAATGACCATCTAAAGCCTGCCCTTCATTGGTCTGGTTCACGTCTCCAAACCAGCT ENSG00000204936: 9057 CD177P1 $19: 43372406$ GAGAAATGGCCATCCAAGCCTGCCCTTCATTGGTCTGGTCATGTCTCCAACCAGCT
19:43372347 CD177 ENSG00000204936: 9058 TGGATGGTAGCAGAGACTTCAGGGTGCTCCAGCCAAACGTATTTGGGCATCACCATGACC ENSG00000204936: 9117 CD177P1
19:43372346 TGGATGGTAGCAGAGACTTCAGGGTCTCCAGCCAACGTATTTGGCATCACCATGACC

CD177 ENSG00000204936: 9118 TGGGAGGGGAGATGCACTGAGACGTATGAGGCTTCCAGCCTAGCAGCCAGGGCCCTAGC ENSG00000204936: 9177 CD177P1 $19: 43372286$ TGGGAGGGAGATGCACTGAGATGTATGAGGCTTCCAGCCTAGCAGCCAGGCCCTAGC
19:43372227

CD177 ENSG00000204936: 9178 ACAAACAGGAGGCTCGCCCATCTGAGCAACTGCAGGAGAGGTTAGTACAGTCATGCATT ENSG00000204936: 9237 I|| || |||| |||| |||||||||||||||| || |||||||||||||||||||||| ||||||||||||| 
CD177 ENSG00000204936: 9238 GCTTAACGACAGGGACGTGTCGTTAGAAATGTGTCGTTAGGTGATTTTATGACCATAGGA ENSG00000204936: 9297 CD177P1 19:43372166 GCTTTACGACAGGACGTGTCGTAGAATGTTCGTTAGGTGTTTTATCACGAAGGA

CD177 ENSG00000204936: 9298 ACATTGTAGCGTGCACTTACACCAACCCAGATGGTACAGCCCAATACACACCCAGGATGG ENSG00000204936: 9357 CD177P1
19:43372106 ACATTGTAGAGTGCACTACACCAACCCAGATGGTACAGCCCAATACACACCCAGGATG

CD177 ENSG00000204936: 9358 ACGCTAGAGTCGACTGCTCCTAGGCTACAAGCCTGCAGTGCATGTTATGGTGTGAATACT ENSG00000204936: 9417 CD177P1
$19: 43372046$ ATGGTAGAGTGACTGCTCCTAGGCTACAAGCCTGCAGTGCATGTTATGGTGGAATACT ENSG00000204936: $\quad 9424$

CD177 ENSG00000204936: 9418 GCAGGCAA

$19: 43371980$

19:43371986 GCAGGCA

CD177 ENSG00000204936: 9426 TCTTAACACCACGGCAAGTATTTGTGCATCTACACACATCTAAACATAGAAAAGGTACAG ENSG00000204936: 9485 CD177P1 19:43371979 TCGTAACACCACGGCAGTATTTGTGATCTATACACATCTAAACATAGAAAGGACAG

CD177 ENSG00000204936: 9486 CATAAATACACTATTGTCATCTCAGCAGA

CD177P1 19:43371919 CATAAATACACTATTGTCATCTCAGGAGA

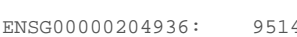

$19: 43371891$ 